J.M. Almira ${ }^{\star} \cdot$ P.J. Torres ${ }^{\star \star}$

\title{
Invariance of the stability of meissner's equation under a permutation of its intervals
}

Received: March 3, 2000

Published online: July 13, 2001 - (C) Springer-Verlag 2001

\begin{abstract}
The aim of this paper is to study a particular aspect of the stability of Meissner's equation, which leads to some interesting questions about the invariance of the trace of a product of matrices.
\end{abstract}

Mathematics Subject Classification (2000). 34C11, 15A99

\section{Introduction}

In 1918, Meissner [7] investigated the second-order linear differential equation

$$
x^{\prime \prime}+\alpha(t) x=0
$$

where $\alpha$ is a $T$-periodic piecewise constant function defined as

$$
\alpha(t)=w_{i} \quad t \in((i-1) h, i h], i=1, \ldots, n,
$$

where $h=T / n$ and $w_{i}>0$ for all $i$. Solutions should be understood in the weak sense, as solutions of the corresponding Volterra equation. In the case of two pieces in $\alpha$ this equation has been studied in detail by Hochstadt [3], who also provides some references about applications to one-dimensional crystal models.

Meissner's equation is a special case of Hill's equation, for which there exists a well-known general theory (see for instance [6]). One of the basic properties of this equation is that either all solutions are unbounded or all solutions are bounded. In the first case, the equation is said to be unstable, while in the second case the equation is said to be stable.

The main question we raise here is the following: Which permutations of the pieces of $\alpha$ preserve the stability character of Meissner's equation?

J.M. Almira: Universidad de Jaén, Departamento de Matemáticas, 23700, Linares, Spain P.J. Torres: International School for Advanced Studies ISAS-SISSA, Via Beirut 2-4, 34013 Trieste, Italy. Permanent address: Universidad de Granada, Departamento de Matemática Aplicada, 18071 Granada, Spain, e-mail: ptorresesissa.it, ptorres@ugr.es

* Partially supported by Junta de Andalucía, Grupo de Investigación Aproximación y Métodos Numéricos, código FQM 0178, e-mail: jmalmira@ujaen. es

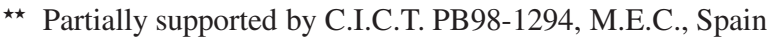


Basically, the stability of a Hill's equation depends on the discriminant, that is, the trace of the monodromy matrix. The advantage of Meisner's equation is that it is integrable: the equation can be solved on each interval as a usual harmonic oscillator, matching the solution at the endpoints by making $x$ and $x^{\prime}$ continuous. Then, it is very easy to verify that the monodromy matrix is

$$
\Phi=A_{n} A_{n-1} \cdots A_{1},
$$

where $A_{i}$ are the exponential matrices

$$
e^{\left(\begin{array}{cc}
0 & 1 \\
-w_{i} & 0
\end{array}\right) h}=\left(\begin{array}{rr}
\cos \left(w_{i} h\right) & \left(1 / w_{i}\right) \sin \left(w_{i} h\right) \\
-w_{i} \sin \left(w_{i} h\right) & \cos \left(w_{i} h\right)
\end{array}\right) .
$$

If the trace of a square matrix $A$ is denoted by $\operatorname{tr}(A)$, it is known that equation (1) is unstable if $|\operatorname{tr}(\Phi)|>2$ and it is stable if $|\operatorname{tr}(\Phi)|<2$. Therefore, our problem is reduced to determining the set of permutations $\sigma \in \mathbf{S}_{n}$ which preserve the trace of a product of matrices

$$
\operatorname{tr}\left(A_{\sigma(1)} A_{\sigma(2)} \cdots A_{\sigma(n)}\right)=\operatorname{tr}\left(A_{1} A_{2} \cdots A_{n}\right)
$$

(for arbitrary matrices $A_{i}$ ).

In such a case we will say that the permutation is admissible. If a permutation $\sigma \in \mathbf{S}_{n}$ is admissible, equation (1) has the same stability character as the equation

$$
x^{\prime \prime}+\alpha_{\sigma}(t) x=0,
$$

where

$$
\alpha_{\sigma}(t)=w_{\sigma(i)} t \in((i-1) h, i h], i=1, \ldots, n .
$$

In this sense, a dynamical problem leads to a basic but non-trivial problem on matrix algebra, which we are going to study below.

As a further remark, it is interesting to note that this question can be understood as a stability problem for a periodically switched system. This topic has generated a considerable number of recent papers (see [1], [8], [5], [9], [2] and their references), mainly because its relationship with control theory in the following way: let us consider

$$
x^{\prime \prime}(t)+\sum_{i=1}^{n} w_{i} u_{i} x(t)=0,
$$

where the admissible controls are periodic "step" functions of the form $u_{i}=1$ in $\left[\left(\sigma_{i}-1\right) h, \sigma_{i} h\right]$ and $u_{i}=0$ outside. Then we look for the set of admissible controls $u_{i}$ that conserve the stability character of (1).

\section{The general problem}

In this section we will give a characterization of the set of admissible permutations we have defined in the above section. It is a well-known (see [4]) fact that $\operatorname{tr}(A B)=$ 
$\operatorname{tr}(B A)$ for all matrices $A, B \in M_{N}(\mathbb{R})$. This does not imply the equality of the traces

$$
\operatorname{tr}\left(A_{\sigma(1)} A_{\sigma(2)} \cdots A_{\sigma(n)}\right)
$$

for arbitrary matrices $A_{i} \in M_{N}(\mathbb{R})$ and arbitrary permutations $\sigma \in S_{n}(n \geq 3$, $N \geq 2$ ). For example,

$$
\left(\begin{array}{ll}
2 & 0 \\
1 & 3
\end{array}\right)\left(\begin{array}{ll}
1 & -1 \\
0 & 1
\end{array}\right)\left(\begin{array}{ll}
1 & -1 \\
1 & 1
\end{array}\right)=\left(\begin{array}{cc}
0 & -4 \\
3 & 1
\end{array}\right)
$$

and

$$
\left(\begin{array}{ll}
2 & 0 \\
1 & 3
\end{array}\right)\left(\begin{array}{ll}
1 & -1 \\
1 & 1
\end{array}\right)\left(\begin{array}{ll}
1 & -1 \\
0 & 1
\end{array}\right)=\left(\begin{array}{ll}
2 & -4 \\
4 & -2
\end{array}\right) .
$$

So, it is of interest to know for which permutations $\sigma$ we can guarantee that

$$
\operatorname{tr}\left(A_{\sigma(1)} A_{\sigma(2)} \cdots A_{\sigma(n)}\right)=\operatorname{tr}\left(A_{1} A_{2} \cdots A_{n}\right)
$$

for arbitrary matrices $A_{i} \in M_{N}(\mathbb{R})$.

We will only consider the case $N=2$, since, if $\operatorname{tr}\left(A_{\sigma(1)} A_{\sigma(2)} \cdots A_{\sigma(n)}\right) \neq$ $\operatorname{tr}\left(A_{1} A_{2} \cdots A_{n}\right)$ for some permutation $\sigma \in S_{n}$ and some matrices $A_{i} \in M_{2}(\mathbb{R})$, then for all $N>2$ we can take

$$
B_{i}=\left(\begin{array}{ll}
A_{i} & \mathbf{0} \\
\mathbf{0} & \mathbf{I}_{N-2}
\end{array}\right), i=1, \ldots, n,
$$

(where $\mathbf{I}_{k}$ denotes the identity matrix of order $k$ for all $k$ ) and it is clear that

$$
\operatorname{tr}\left(B_{\sigma(1)} B_{\sigma(2)} \cdots B_{\sigma(n)}\right) \neq \operatorname{tr}\left(B_{1} B_{2} \cdots B_{n}\right) .
$$

Theorem 1. Let $n \geq 3$ be fixed. Then $\sigma \in S_{n}$ satisfy

$$
\operatorname{tr}\left(A_{\sigma(1)} A_{\sigma(2)} \cdots A_{\sigma(n)}\right)=\operatorname{tr}\left(A_{1} A_{2} \cdots A_{n}\right)
$$

for arbitrary matrices $A_{i} \in M_{N}(\mathbb{R})$ if and only if

$$
\sigma \in \operatorname{span}\{\boldsymbol{\tau}\}=\left\{\mathbf{I d}, \boldsymbol{\tau}, \boldsymbol{\tau}^{2}, \ldots, \boldsymbol{\tau}^{n-1}\right\},
$$

where $\tau$ is the $n$-cycle $\tau=\left(\begin{array}{llll}1 & 2 & 3 & \cdots\end{array}\right)$.

Proof. First of all, we note that the set

$$
G=\left\{\sigma \in S_{n}: \operatorname{tr}\left(A_{\sigma(1)} A_{\sigma(2)} \cdots A_{\sigma(n)}\right)=\operatorname{tr}\left(A_{1} A_{2} \cdots A_{n}\right) \text { for all } A_{i} \in M_{N}(\mathbb{R})\right\}
$$

is a subgroup of $S_{n}$. This follows from the fact that if $\sigma_{1}, \sigma_{2} \in G$, then

$$
\begin{aligned}
\operatorname{tr}\left(A_{\sigma_{2}\left(\sigma_{1}(1)\right)} A_{\sigma_{2}\left(\sigma_{1}(2)\right)} \cdots A_{\sigma_{2}\left(\sigma_{1}(n)\right)}\right) & =\operatorname{tr}\left(A_{\sigma_{1}(1)} A_{\sigma_{1}(2)} \cdots A_{\sigma_{1}(n)}\right) \\
& =\operatorname{tr}\left(A_{1} A_{2} \cdots A_{n}\right)
\end{aligned}
$$


for arbitrary matrices $A_{i} \in M_{N}(\mathbb{R})$ and that all elements of $S_{n}$ are of finite order. What we want to prove is that $G=\operatorname{span}\{\tau\}$, where $\boldsymbol{\tau}(k)=k+1$ for all $k<n$ and $\tau(n)=1$.

It is clear that $\operatorname{span}\{\tau\}$ is a subset of $G$ since

$$
\begin{aligned}
\operatorname{tr}\left(A_{1}\left(A_{2} \cdots A_{n}\right)\right) & =\operatorname{tr}\left(\left(A_{2} \cdots A_{n}\right) A_{1}\right) \\
& =\operatorname{tr}\left(A_{\tau(1)} A_{\tau(2)} \cdots A_{\boldsymbol{\tau}(n)}\right)
\end{aligned}
$$

for arbitrary matrices $A_{i} \in M_{N}(\mathbb{R})$.

On the other hand, let us assume that $\sigma \notin \operatorname{span}\{\tau\}$. Let $p=\sigma(1)$. Then $\boldsymbol{\tau}^{n-p+1} \sigma(1)=\boldsymbol{\tau}^{n-p+1}(p)=1$. Now, it is clear that $\boldsymbol{\tau}^{n-p+1} \sigma \neq$ Id since $\sigma \notin$ $\operatorname{span}\{\boldsymbol{\tau}\}$. Let $i_{0}$ be the least integer such that $\boldsymbol{\tau}^{n-p+1} \sigma(i) \neq i$ and let $j_{0}=$ $\tau^{n-p+1} \sigma\left(i_{0}\right)$. Then $j_{0}>i_{0}$ and $\tau^{n-p+1} \sigma$ is of the form

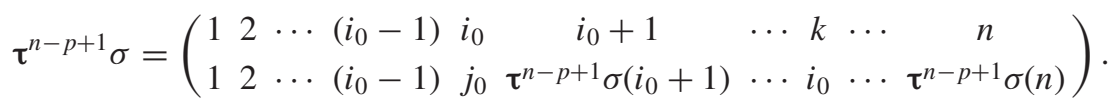

Let $A, B, C \in M_{2}(\mathbb{R})$ be such that $\operatorname{tr}(A B C) \neq \operatorname{tr}(A C B)$ and set $A_{1}=A$, $A_{i_{0}}=B, A_{j_{0}}=C$, and $A_{k}=\mathbf{I}_{N}$ for all $k \notin\left\{1, i_{0}, j_{0}\right\}$. Then

$$
\begin{aligned}
\operatorname{tr}(A C B) & =\operatorname{tr}\left(A_{\tau^{n-p+1} \sigma(1)} A_{\tau^{n-p+1} \sigma(2)} A_{\tau^{n-p+1} \sigma(3)} \cdots A_{\tau^{n-p+1} \sigma(n)}\right) \\
& =\operatorname{tr}\left(A_{\tau^{p-1}\left(\tau^{n-p+1} \sigma(1)\right)} A_{\tau^{p-1}\left(\tau^{n-p+1} \sigma(2)\right)} \cdots A_{\tau^{p-1}\left(\tau^{n-p+1} \sigma(n)\right)}\right) \\
& =\operatorname{tr}\left(A_{\sigma(1)} A_{\sigma(2)} \cdots A_{\sigma(n)}\right)
\end{aligned}
$$

and

$$
\operatorname{tr}\left(A_{1} A_{2} A_{3} \cdots A_{n}\right)=\operatorname{tr}(A B C) \neq \operatorname{tr}(A C B)=\operatorname{tr}\left(A_{\sigma(1)} A_{\sigma(2)} \cdots A_{\sigma(n)}\right)
$$

so that $\sigma \notin G$.

\section{Main result}

We have solved the problem of equality of the trace of a product for arbitrary matrices but we have not solved the problem for the set of matrices which appear in the study of the resonance of the Meissner's equation. For instance, if $\mathbf{x}(t)$ is solution of $\mathbf{x}^{\prime \prime}+\alpha(t) \mathbf{x}=0$ then $\mathbf{x}(-t)$ is solution of $\mathbf{x}^{\prime \prime}+\alpha(-t) \mathbf{x}=0$ and, in consequence both equations have the same eigenvalues. Moreover, the monodromy matrix of the second equation is

$$
\Psi(T)=A_{1} A_{2} \cdots A_{n},
$$

where $\Phi(T)=A_{n} A_{n-1} \ldots A_{1}$ is the monodromy matrix of the original equation. Hence

$$
\operatorname{tr}\left(A_{1} A_{2} A_{3} \cdots A_{n}\right)=\operatorname{tr}\left(A_{n} A_{n-1} A_{n-2} \cdots A_{1}\right)
$$

for all matrices $A_{i}$ of the form

$$
A_{i}=\left(\begin{array}{ll}
\cos \left(w_{i} h\right) & \left(1 / w_{i}\right) \sin \left(w_{i} h\right) \\
-w_{i} \sin \left(w_{i} h\right) & \cos \left(w_{i} h\right)
\end{array}\right), w_{i}, h>0
$$


This is not in contradiction with Theorem 1. It only claims that the set of permutations which preserve the trace of a product of matrices of the form (3) is bigger than $\operatorname{span}\{\tau\}$. In what follows, we will denote by $\mathcal{N}$ the set of all matrices of the form (3) and by $\mathbf{D}_{n}$ the set

$$
\begin{aligned}
\mathbf{D}_{n}= & \left\{\sigma \in \mathbf{S}_{n}: \operatorname{tr}\left(A_{\sigma(1)} A_{\sigma(2)} \cdots A_{\sigma(n)}\right)=\operatorname{tr}\left(A_{1} A_{2} \cdots A_{n}\right)\right. \\
& \text { for arbitrary matrices } \left.A_{i} \in \mathcal{N}\right\}
\end{aligned}
$$

It is clear that $\operatorname{span}\{\tau, \zeta\}$ is a subset of $\mathbf{D}_{n}$, where $\tau$ is the n-cycle $\tau=(12 \cdots n)$ and $\zeta(k)=n-k+1$ for all $k$. Furthermore, if $n \leq 3$ then $\operatorname{span}\{\boldsymbol{\tau}, \zeta\}=\mathbf{D}_{n}=\mathbf{S}_{n}$. In what follows we will assume that $n \geq 4$.

Now we can write the main result of this section, which is the following:

Theorem 2. $\mathbf{D}_{n}=\operatorname{span}\{\boldsymbol{\tau}, \zeta\}$. Moreover, $\mathbf{D}_{n}$ is a group with the composition of functions as operation, which is isomorphic to the diedral group of order $n$ (and this justifies our notation).

To prove this theorem, we will first prove the lemmas:

Lemma 3. $\operatorname{span}\{\tau, \zeta\}$ is isomorphic to the diedral group of order $n$.

Proof. It follows from the fact that $\tau$ is an n-cycle that it has order $n$. On the other hand,

$$
\zeta^{2}(k)=\zeta(n-k+1)=n-n+k-1+1=k
$$

for all $k$, so that $\zeta$ is an element of order two. Hence we only need to prove that the relation $\zeta \tau=\tau^{n-1} \zeta$ holds. Now, taking into consideration that

$$
\tau^{n-1}=\tau^{-1}=\left(\begin{array}{llllc}
1 & 2 & 3 & \cdots & n \\
n & 1 & 2 & \cdots & n-1
\end{array}\right),
$$

it is easy to check the relation

$$
\tau^{n-1} \zeta=\left(\begin{array}{ccccc}
1 & 2 & 3 & \cdots & n \\
n-1 & n-2 & n-3 & \cdots & n
\end{array}\right)=\zeta \tau .
$$

Lemma 4. Let us denote by $\mathbf{A}$ and $\mathbf{B}$ the matrices

$$
\mathbf{A}=\left(\begin{array}{ll}
\cos (\pi / n) & \sin (\pi / n) \\
-\sin (\pi / n) & \cos (\pi / n)
\end{array}\right) ; \mathbf{B}=\left(\begin{array}{ll}
0 & 2 / n \\
-n / 2 & 0
\end{array}\right)
$$

where $n$ is supposed to be an integer $n \geq 4$, and let $p, q \in\{1, \ldots, n-3\}$ be such that $p+q=n-2$. Then

$$
\operatorname{tr}\left(\mathbf{A}^{n-2} \mathbf{B}^{2}\right) \neq \operatorname{tr}\left(\mathbf{A}^{p} \mathbf{B} \mathbf{A}^{q} \mathbf{B}\right)
$$


Proof. To compute these traces we will take in consideration the relations $\mathbf{B}^{2}=-\mathbf{I}$ and

$$
\mathbf{A}^{k}=\left(\begin{array}{ll}
\cos (k \pi / n) & \sin (k \pi / n) \\
-\sin (k \pi / n) & \cos (k \pi / n)
\end{array}\right)
$$

for all integer $k$. Hence

$$
\begin{aligned}
\operatorname{tr}\left(\mathbf{A}^{n-2} \mathbf{B}^{2}\right) & =-\operatorname{tr}\left(\mathbf{A}^{n-2}\right)=-2 \cos ((n-2) \pi / n) \\
& =-2 \cos (p \pi / n) \cos (q \pi / n)+2 \sin (p \pi / n) \sin (q \pi / n)
\end{aligned}
$$

and

$\operatorname{tr}\left(\mathbf{A}^{p} \mathbf{B} \mathbf{A}^{q} \mathbf{B}\right)=-2 \cos (p \pi / n) \cos (q \pi / n)+\left(n^{2} / 4+4 / n^{2}\right) \sin (p \pi / n) \sin (q \pi / n)$.

Now it is clear that these two traces are different because $\sin (p \pi / n) \sin (q \pi / n)$ $\neq 0$, since $p, q \neq \dot{n}$, and the unique integer solution of $n^{2} / 4+4 / n^{2}-2=0$ is $n=2$ (but we have set $n \geq 4$ ).

Proof of the main result. We only need to prove that $\mathbf{D}_{n}$ is a subset of $\operatorname{span}\{\tau, \zeta\}$. To do this, it will be enough to prove that for all $\sigma \in \mathbf{S}_{n} \backslash \operatorname{span}\{\tau, \zeta\}$ there are matrices $A_{i} \in \mathcal{N}, i=1, \ldots, n$ such that

$$
\operatorname{tr}\left(A_{\sigma(1)} A_{\sigma(2)} \cdots A_{\sigma(n)}\right) \neq \operatorname{tr}\left(A_{1} A_{2} \cdots A_{n}\right) .
$$

There is no loss of generality in assuming that $\sigma(1)=1$, since we can compose with arbitrary elements of $\operatorname{span}\{\boldsymbol{\tau}\}$ without perturbing the $\operatorname{trace} \operatorname{tr}\left(A_{\sigma(1)} A_{\sigma(2)}\right.$ $\left.\cdots A_{\sigma(n)}\right)$.

Let $k=\max \{i: \sigma(i)<i\}$. It is clear that $k>2$ and that $\sigma_{\mid\{k+1, \ldots, n\}}=$ $1_{\{\{k+1, \ldots, n\}}$. We make the following cases:

Case 1. $k<n$. Then there exists some $t \in\{2, \ldots, k-1\}$ such that $\sigma(t)=k$ and taking

$$
A_{i}=\left\{\begin{array}{l}
\mathbf{B} \quad i \in\{k, k+1\} \\
\mathbf{A} \text { otherwise }
\end{array}\right.
$$

we have that

$$
A_{\sigma(i)}= \begin{cases}\mathbf{B} & i \in\{t, k+1\} \\ \mathbf{A} & \text { otherwise }\end{cases}
$$

where $\mathbf{A}, \mathbf{B}$ are defined in Lemma 4. Hence

$$
\operatorname{tr}\left(A_{1} A_{2} \cdots A_{n}\right)=\operatorname{tr}\left(\mathbf{A}^{k-1} \mathbf{B}^{2} \mathbf{A}^{n-k-1}\right)=\operatorname{tr}\left(\mathbf{A}^{n-2} \mathbf{B}^{2}\right)
$$

and

$$
\operatorname{tr}\left(A_{\sigma(1)} A_{\sigma(2)} \cdots A_{\sigma(n)}\right)=\operatorname{tr}\left(\mathbf{A}^{t-1} \mathbf{B} \mathbf{A}^{k-t+1} \mathbf{B A}^{n-2-k}\right)=\operatorname{tr}\left(\mathbf{A}^{p} \mathbf{B} \mathbf{A}^{q} \mathbf{B}\right)
$$

where $p+q=n-2, p=n+t-k-3$, and $q=k-t+1$. It follows from Lemma 4 that these two traces are different. 
Case 2. $k=n$. Then there exists some $t \in\{2, \ldots, n-1\}$ such that $\sigma(t)=n$. If $t=2$, then

$$
\begin{aligned}
\operatorname{tr}\left(A_{\sigma(1)} A_{\sigma(2)} \cdots A_{\sigma(n)}\right) & =\operatorname{tr}\left(A_{1} A_{n} A_{\sigma(2)} \cdots A_{\sigma(n)}\right) \\
& =\operatorname{tr}\left(A_{\sigma(n)} \cdots A_{\sigma(2)} A_{n} A_{1}\right) \\
& =\operatorname{tr}\left(A_{1} A_{\sigma(n)} \cdots A_{\sigma(2)} A_{n}\right) \\
& =\operatorname{tr}\left(A_{\theta(1)} A_{\theta(2)} \cdots A_{\theta(n)}\right)
\end{aligned}
$$

for a certain $\theta \in \mathbf{S}_{n} \backslash \operatorname{span}\{\boldsymbol{\tau}, \zeta\}$ which is in the first case. If $t>2$, taking

$$
A_{i}= \begin{cases}\mathbf{B} & i \in\{1, n\} \\ \mathbf{A} & \text { otherwise }\end{cases}
$$

we have that

$$
A_{\sigma(i)}= \begin{cases}\mathbf{B} & i \in\{1, t\} \\ \mathbf{A} & \text { otherwise }\end{cases}
$$

Hence

$$
\operatorname{tr}\left(A_{1} A_{2} \cdots A_{n}\right)=\operatorname{tr}\left(\mathbf{B} \mathbf{A}^{n-2} \mathbf{B}\right)=\operatorname{tr}\left(\mathbf{A}^{n-2} \mathbf{B}^{2}\right)
$$

and

$$
\operatorname{tr}\left(A_{\sigma(1)} A_{\sigma(2)} \cdots A_{\sigma(n)}\right)=\operatorname{tr}\left(\mathbf{B} \mathbf{A}^{p} \mathbf{B} \mathbf{A}^{q}\right)=\operatorname{tr}\left(\mathbf{A}^{p} \mathbf{B} \mathbf{A}^{q} \mathbf{B}\right),
$$

for certain integers $p, q$ such that $p+q=n-2$. Hence we may use Lemma 4 in this case to prove that the traces are different.

Once we have proved this result, a natural question arises: it has been proved that permutations not belonging to $\operatorname{span}\{\tau, \zeta\}$ change the discriminant, but the stability of Meissner's equation can still be conserved if this trace remains belonging to the adequate interval. We try to answer this question with the following theorem and corollary:

Theorem 5. Let us assume that $\max \{p, q\} \geq 2$ and let $n=p+q+2$. Then there are matrices $\mathbf{C}, \mathbf{D} \in \mathcal{N}$ such that

$$
\left|\operatorname{tr}\left(\mathbf{C}^{n-2} \mathbf{D}^{2}\right)\right|<2<\operatorname{tr}\left(\mathbf{C}^{p} \mathbf{D C}^{q} \mathbf{D}\right)
$$

Proof. Take $\mathbf{C}=\left(\begin{array}{ll}\cos \left(\pi / n^{2}\right) & \sin \left(\pi / n^{2}\right) \\ -\sin \left(\pi / n^{2}\right) & \cos \left(\pi / n^{2}\right)\end{array}\right)$ and $\mathbf{D}=\left(\begin{array}{ll}0 & 2 / n^{2} \\ -n^{2} / 2 & 0\end{array}\right)$. Then it is easy to check that (where we need to use that $\max \left\{p \pi / n^{2}, q \pi / n^{2}\right\} \leq \frac{\pi}{4}$, that is: $n^{2} \geq 4 \max \{p, q\}$, which obviously follows from the relation $p+q=n-2$ ),

$$
\begin{aligned}
\operatorname{tr}\left(\mathbf{C}^{p} \mathbf{D} \mathbf{C}^{q} \mathbf{D}\right) & =\left(n^{4} / 4+4 / n^{4}\right) \sin \left(p \pi / n^{2}\right) \sin \left(q \pi / n^{2}\right)-2 \cos \left(p \pi / n^{2}\right) \cos \left(q \pi / n^{2}\right) \\
& \geq\left(n^{4} / 4+4 / n^{4}\right) \sin \left(p \pi / n^{2}\right) \sin \left(q \pi / n^{2}\right)-2 \\
& \geq\left(n^{4} / 4+4 / n^{4}\right) 8 p q / n^{4}-2 \\
& =2(p q-1)+32 p q / n^{8}>2(\text { since } p q-1 \geq 1) .
\end{aligned}
$$

On the other hand, $\left|\operatorname{tr}\left(\mathbf{C}^{n-2} \mathbf{D}^{2}\right)\right|<2$ is trivial. 
Corollary 6. For all $\sigma \in \mathbf{S}_{n} \backslash \mathbf{s p a n}\{\tau, \zeta\}$ there exists a Meissner's equation $\mathbf{x}^{\prime \prime}+$ $\alpha \mathbf{x}=\mathbf{0}$, such that

$$
\left|\operatorname{tr}\left(\boldsymbol{\Phi}_{\alpha}(T)\right)\right|<2<\left|\operatorname{tr}\left(\Phi_{\alpha_{\sigma}}(T)\right)\right|
$$

where $T$ is the period of $\alpha$.

Proof. We have shown that for all $\sigma \in \mathbf{S}_{n} \backslash \operatorname{span}\{\boldsymbol{\tau}, \zeta\}$ there exists a Meissner's equation $\mathbf{x}^{\prime \prime}+\alpha \mathbf{x}=\mathbf{0}$, where $\alpha$ is a piecewise constant periodic function which only takes two values $\left\{w_{1}, w_{2}\right\}$ and such that

$$
\operatorname{tr}\left(\Phi_{\alpha}(T)\right)=\operatorname{tr}\left(\mathbf{X}^{n-2} \mathbf{Y}^{2}\right) \text {, and } \operatorname{tr}\left(\Phi_{\alpha_{\sigma}}(T)\right)=\operatorname{tr}\left(\mathbf{X}^{p} \mathbf{Y} \mathbf{X}^{q} \mathbf{Y}\right)
$$

(for certain integers $p, q$ with $p+q=n-2$ which depend of $\sigma$ ), where

$$
\begin{aligned}
& \mathbf{X}=\left(\begin{array}{ll}
\cos \left(w_{1} T / n\right) & \left(1 / w_{1}\right) \sin \left(w_{1} T / n\right) \\
-w_{1} \sin \left(w_{1} T / n\right) & \cos \left(w_{1} T / n\right)
\end{array}\right), \\
& \mathbf{Y}=\left(\begin{array}{ll}
\cos \left(w_{2} T / n\right) & \left(1 / w_{2}\right) \sin \left(w_{2} T / n\right) \\
-w_{2} \sin \left(w_{2} T / n\right) & \cos \left(w_{2} T / n\right)
\end{array}\right),
\end{aligned}
$$

$T$ is the period of $\alpha$ and $n$ is the number of pieces used in the definition of $\alpha$.

If $\max \{p, q\}>1$, then we can take $w_{1}=1, w_{2}=n^{2} / 2$ and $T=\pi / n$, so that $\mathbf{X}=\mathbf{C}$ and $\mathbf{Y}=\mathbf{D}$ in the proof of Theorem 5, and $\left|\operatorname{tr}\left(\boldsymbol{\Phi}_{\alpha}(T)\right)\right|<2<$ $\left|\operatorname{tr}\left(\boldsymbol{\Phi}_{\alpha_{\sigma}}(T)\right)\right|$.

If $p=q=1$ then $n=4$ and we need to choose the matrices $\mathbf{X}, \mathbf{Y} \in \mathcal{N}$ in order to satisfy

$$
\left|\operatorname{tr}\left(\mathbf{X}^{2} \mathbf{Y}^{2}\right)\right|<2<|\operatorname{tr}(\mathbf{X Y X Y})|
$$

We set

$$
\begin{aligned}
\mathbf{X} & =\left(\begin{array}{ll}
\cos ((2+1 / 4) \pi) & \frac{1}{2+1 / 4} \sin ((2+1 / 4) \pi) \\
-(2+1 / 4) \sin ((2+1 / 4) \pi) & \cos ((2+1 / 4) \pi)
\end{array}\right) \\
& =\left(\begin{array}{ll}
\sqrt{2} / 2 & 2 \sqrt{2} / 9 \\
-9 \sqrt{2} / 8 & \sqrt{2} / 2
\end{array}\right) \\
\mathbf{Y} & =\left(\begin{array}{ll}
\cos (\pi / 2) & 2 \sin (\pi / 2) \\
-(1 / 2) \sin (\pi / 2) & \cos (\pi / 2)
\end{array}\right)=\left(\begin{array}{ll}
0 & 2 \\
-1 / 2 & 0
\end{array}\right),
\end{aligned}
$$

and now it is easy to check that $\operatorname{tr}(\mathbf{X Y X Y})=9.1497>2$ and $\operatorname{tr}\left(\mathbf{X}^{2} \mathbf{Y}^{2}\right)=0<2$.

Acknowledgements. The authors would like to express their gratitude to N. Del Toro and R. Ortega for their valuable comments and suggestions. 


\section{References}

1. Agrachev, A.A., Liberzon, D.: Lie-algebraic conditions for exponential stability of switched systems. In press

2. Branicky, M.: Multiple Lyapunov functions and other analysis tools for switched and hybrid systems. IEEE Trans. Automat. Control 43, 4, 475-482 (1998)

3. Hochstadt, H.: A special Hill's equation with discontinuous coefficients. Am. Math. Monthly 70, 18-26 (1963)

4. Lang, S.: Linear Algebra. Reading, Massachusets: Addison-Wesley 1980

5. Liberzon, D., Hespanna, J.P., Morse, A.S.: Stability of switched systems: a Lie-algebraic condition. Systems Control Lett. 37, 117-122 (1999)

6. Magnus, W., Winkler, S.: Hill's equation. New York: Dover Publ. Comp. 1966

7. Meissner, E.: Ueber Schuettelschwingungen in Systemen mit periodisch veraenderlicher Elastizitaet. Schweizer Bauzeitung 72, 95-98 (1918)

8. Sarychev, A.V.: High order averaging and stability of time-varing systems. In press

9. Tokarzewski, J.: Stability of periodically switched linear systems and the switching frequency. Internat. J. Systems Sci. 18, 4, 697-726 (1987) 